\title{
Density of States Scaling at the Semimetal to Metal Transition in Three Dimensional Topological Insulators
}

\author{
Koji Kobayashi ${ }^{1}$, Tomi Ohtsuki ${ }^{1}$, Ken-Ichiro Imura ${ }^{2,4}$, and Igor F. Herbut ${ }^{3,4}$ \\ ${ }^{1}$ Department of Physics, Sophia University, Chiyoda-ku, Tokyo, 102-8554, Japan \\ ${ }^{2}$ Department of Quantum Matter, AdSM, Hiroshima University, Higashi-Hiroshima, 739-8530, Japan \\ ${ }^{3}$ Department of Physics, Simon Fraser University, Burnaby, British Columbia, V5A 1S6, Canada and \\ ${ }^{4}$ Max-Planck-Institut für Physik komplexer Systeme, Nöthnitzer Str. 38, 01187 Dresden, Germany
}

(Dated: November 5, 2018)

\begin{abstract}
The quantum phase transition between the three dimensional Dirac semimetal and the diffusive metal can be induced by increasing disorder. Taking the system of disordered $\mathbb{Z}_{2}$ topological insulator as an important example, we compute the single particle density of states by the kernel polynomial method. We focus on three regions: the Dirac semimetal at the phase boundary between two topologically distinct phases, the tricritical point of the two topological insulator phases and the diffusive metal, and the diffusive metal lying at strong disorder. The density of states obeys a novel single parameter scaling, collapsing onto two branches of a universal scaling function, which correspond to the Dirac semimetal and the diffusive metal. The diverging length scale critical exponent $\nu$ and the dynamical critical exponent $z$ are estimated, and found to differ significantly from those for the conventional Anderson transition. Critical behavior of experimentally observable quantities near and at the tricritical point is also discussed.
\end{abstract}

PACS numbers: $71.30 .+\mathrm{h}$, 05.70.Jk, 71.23.-k, 71.55.Ak

Topological classification of different insulating phases [1, 2] is an emerging new paradigm in condensed matter physics. Unlike in the Landau theory of phase transitions that is rooted in the idea of spontaneous breaking of symmetry [3], it is less clear how to describe different universality classes of the transitions between topologically different phases. This is because the usual notion of the local order parameter characterizing the different phases is often lacking. At the transition between topologically distinct phases, on the other hand, the gap closes, and the system becomes a semimetal. In three dimensions (3D) such a critical phase is stable in presence of weak disorder [4], but as disorder is increased it gives way to a diffusive metallic state [5]. This transition belongs to a distinct universality class that exhibits nontrivial dynamical and diverging length scale exponents $z$ and $\nu$, for example [5, 6]. The 3D Dirac Hamiltonian in presence of disorder is ubiquitous: it applies to certain phases of superfluid ${ }^{3} \mathrm{He}$ 7], degenerate semiconductors [5], and to the Weyl semimetals [8 11]. Related theories of disordered critical points for two-dimensional interacting Dirac fermions and bosons were also advanced in the past [12, 13].

In this paper we discuss how this disorder-induced fermionic criticality is reflected in the scaling behavior of a readily available physical quantity, the single particle density of states (DOS), which can be understood as a proper order parameter that characterizes such a transition. We then express the critical behavior of Dirac electron velocity, diffusion coefficient, conductivity and anomalous diffusion exponent in terms of $z$ and $\nu$. Such a surprisingly simple description is contrasted with the conventional Anderson transition [14 16], where the DOS remains smooth through the transition.

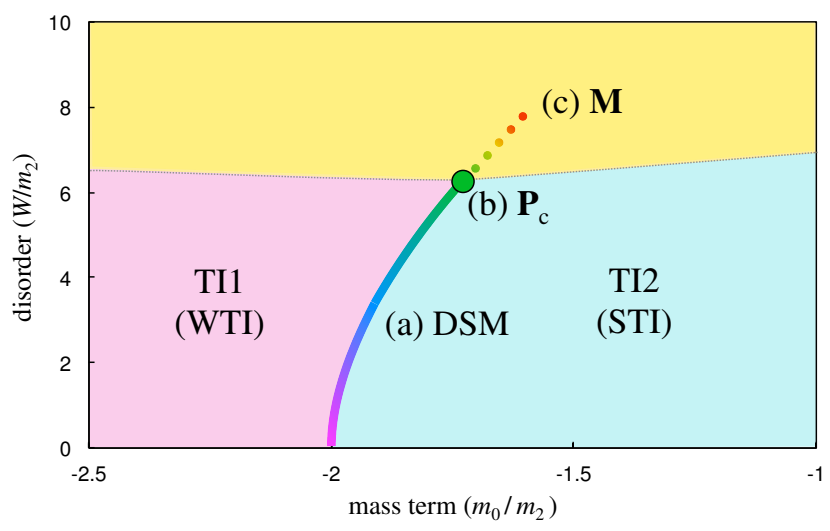

FIG. 1: (Color online) Typical phase diagram of the system under consideration. TI1 and TI2 correspond, respectively, to weak and strong topological insulator (WTI and STI), and DSM to the critical Dirac semimetal phase. The dotted line in the diffusive metal (M) phase (c) is an extrapolation [17] of the DSM line (a). The tricritical point (b) is denoted as $\mathbf{P}_{\mathrm{c}}$.

In order to produce and control the semimetallic phase, we focus on a $3 \mathrm{D}$ time-reversal symmetric topological insulator under disorder. The $\mathbb{Z}_{2}$ topological insulator is interesting in itself, and has lately been a subject of intense theoretical and experimental research, with a number of real material realizations [18]. Consider the phase diagram of a system exhibiting both weak and strong topological insulators (WTI and STI) as some parameter is varied [19 21] (see Fig. 11). In three spatial dimensions disorder is irrelevant in the renormalization group sense, so that at weak disorder a direct transition between two topologically distinct insulating phases [4], say, between TI1 and TI2, remains. (In the specific situation we consider below, TI $1=\mathrm{WTI}$ and TI2 $=$ STI.) Only above 
a finite strength of disorder $W>0$, does the bulk energy gap become completely filled with impurity levels, so that the insulating phases are replaced by a diffusive metallic (M) phase [22] (see Fig. 11). Since TI1 and TI2 are characterized by a different topological number protected by the bulk energy gap, at the phase boundary the bulk spectrum is in general closed. In the present case the system is also protected by time-reversal symmetry, and such a gap closing appears as a (Kramers) degenerate pair of point nodes, i.e., as the Dirac semimetal (DSM) 23] line in the phase diagram. As disorder is increased the DSM line also terminates at the intersection with the insulator-metal phase boundary. In the following we focus on the evolution of the DOS as one moves along the DSM line, through the tricritical point $\mathbf{P}_{\mathrm{c}}$ where the DSM line terminates, and finally reaches inside the metallic phase.

We have previously established, by a detailed numerical study of the conductance 22], that although disorder $W$ shifts the position of the phase boundary [24 29] (determined, e.g., by the position of the conductance peak), it is nevertheless irrelevant; the peak height of the conductance on the DSM line is not influenced by the disorder strength. It was also found [22] that on the DSM line the DOS remains a quadratic function of low energies, exactly as in the clean limit [see the curves (a) in Fig. 2. Whereas the quadratic behavior is left intact by disorder, the coefficient of the quadratic term, which is related to the velocity $v$ of Dirac electrons, is renormalized [30], as in Eq. (21) below.

In this Letter we further quantify the behavior of the DOS on the DSM line toward the diffusive metal phase, and demonstrate that the DOS obeys a single parameter scaling typical of second order phase transitions, with new values of critical exponents. Our analysis is based on the single parameter scaling hypothesis, which is substantially supported by numerical results. The scaling behavior of the DOS is studied using the kernel polynomial method (KPM) 31.

The $3 \mathrm{D}$ disordered $\mathbb{Z}_{2}$ topological insulator is modeled as a Wilson-Dirac-type tight-binding Hamiltonian with an effective momentum-dependent mass term [32],

$$
m(\boldsymbol{k})=m_{0}+m_{2} \sum_{\mu=x, y, z}\left(1-\cos k_{\mu}\right),
$$

implemented on a cubic lattice. The topological nature of the model is controlled by the ratio of two mass parameters $m_{0}$ and $m_{2}$ such that an STI phase with $\mathbb{Z}_{2}$ (one strong and three weak) indices [19 21] $\left(\nu_{0}, \nu_{1} \nu_{2} \nu_{3}\right)=$ $(1,000)$ appears when $-2<m_{0} / m_{2}<0$, while the regime of parameters: $-4<m_{0} / m_{2}<-2$ falls on a WTI phase with $\left(\nu_{0}, \nu_{1} \nu_{2} \nu_{3}\right)=(0,111)$ (see Fig. 1).

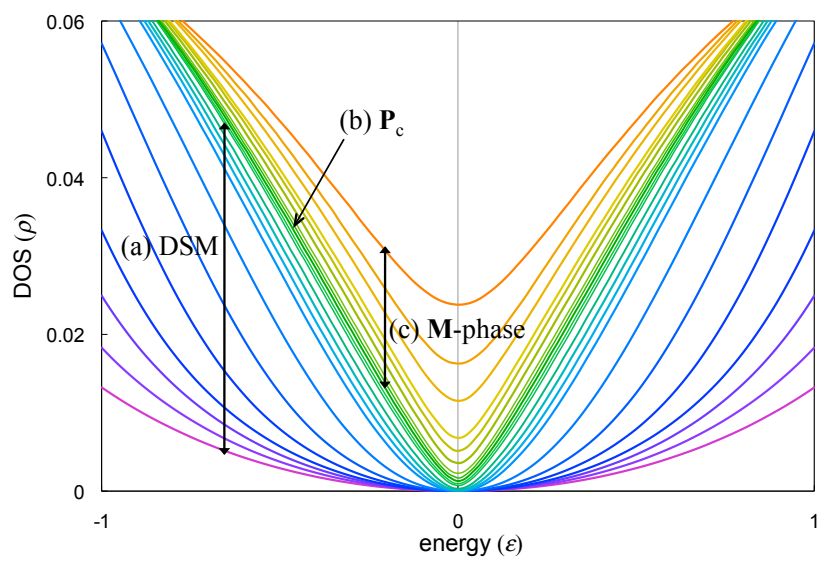

FIG. 2: (Color online) Density of states calculated at different points of the phase diagram $(2 \leq W \leq 7.5)$; (a) on the WTI/STI boundary, (b) at the tricritical point, and (c) in the $\mathbf{M}$-phase. Its energy dependence $\rho(\epsilon)$ is quadratic on the WTI/STI boundary (a), becoming almost linear at the tricritical point (b), while it acquires a finite value $\rho(0)$ at $\epsilon=0$ on the M-side (c). We emphasize that these DOSs are not of the surface, but of the bulk.

In real space our tight-binding Hamiltonian reads

$$
\begin{aligned}
H= & \sum_{\boldsymbol{r}} \sum_{\mu=x, y, z}\left[\left|\boldsymbol{r}+\boldsymbol{e}_{\mu}\right\rangle\left(\frac{\mathrm{i} t}{2} \gamma_{\mu}-\frac{m_{2}}{2} \gamma_{0}\right)\langle\boldsymbol{r}|+\text { h.c. }\right] \\
& +\sum_{\boldsymbol{r}}|\boldsymbol{r}\rangle\left[\left(m_{0}+3 m_{2}\right) \gamma_{0}+V_{\boldsymbol{r}} 1_{4}\right]\langle\boldsymbol{r}|
\end{aligned}
$$

where $\boldsymbol{e}_{\mu}$ is a unit vector in the $\mu$-direction, and $1_{4}$ represents the $4 \times 4$ identity matrix. $\gamma_{\mu}$ and $\gamma_{0}$ form a set of $\gamma$-matrices in a $4 \times 4$ representation,

$$
\gamma_{\mu}=\left(\begin{array}{cc}
0 & \sigma_{\mu} \\
\sigma_{\mu} & 0
\end{array}\right), \gamma_{0}=\left(\begin{array}{cc}
1_{2} & 0 \\
0 & -1_{2}
\end{array}\right)
$$

where $\sigma_{\mu}$ are Pauli matrices and $1_{2}$ is $2 \times 2$ identity matrix. $m_{0}, m_{2}$ and $t$ are mass and hopping parameters, and $V_{\boldsymbol{r}}$ represents a potential disorder distributed uniformly and independently between $-W / 2$ and $W / 2$.

For simplicity, we have assumed the Hamiltonian Eq. (2) to be isotropic. In the actual computation we set the mass and hopping parameters to $m_{2}=1, t=2$. The linear size of the system $L$ is taken to be 200 times the lattice constant, which is enough to reach the thermodynamic limit of DOS per unit volume. We also take the average over two samples, although the statistical error is already sufficiently small for $L=200$, because of the self-averaging nature of DOS. The order of the Chebyshev expansion in KPM is typically a few thousand, so that the DOS becomes smooth. The periodic boundary conditions are imposed on each direction.

The scaling form of the density of states per volume near the Dirac point may be derived as follows. Begin with a dimensionless quantity, the number of states $N(\epsilon, L)$ below the energy $\epsilon$ in the system of size $L$ in $d$ 
dimensions, and assume that it is a function of dimensionless parameters $L / \xi$ and $\epsilon / \epsilon_{0}$,

$$
N(\epsilon, L)=F\left(L / \xi, \epsilon / \epsilon_{0}\right),
$$

where $\xi$ is the characteristic length scale and $\epsilon_{0}$ is the characteristic energy scale. They are related via the dynamical exponent $z$,

$$
\epsilon_{0} \propto \xi^{-z}
$$

Since the number of states should be proportional to $L^{d}$, the above scaling form should be

$$
N(\epsilon, L)=(L / \xi)^{d} f\left(\epsilon \xi^{z}\right) .
$$

From $N(\epsilon, L)$, the DOS per volume $\rho(\epsilon)$ is calculated as

$$
\rho(\epsilon)=\frac{1}{L^{d}} \frac{d N(\epsilon, L)}{d \epsilon},
$$

so that we finally obtain its scaling form,

$$
\rho(\epsilon)=\rho(-\epsilon)=\xi^{z-d} f^{\prime}\left(|\epsilon| \xi^{z}\right) .
$$

The first equality comes from the symmetry of DOS about $\epsilon=0$. Upon introducing the distance from the tricritical point $\delta=\left|W-W_{\mathrm{c}}\right| / W_{\mathrm{c}}$, we may assume that the length scale $\xi$ diverges near the tricritical point $\mathbf{P}_{\mathrm{c}}$ as,

$$
\xi \sim \delta^{-\nu}
$$

where $\nu$ is the critical exponent. Around $\mathbf{P}_{\mathrm{c}}$, the scaling law, Eq. (8), therefore reads,

$$
\rho(\epsilon) \sim \delta^{(d-z) \nu} f^{\prime}\left(|\epsilon| \delta^{-z \nu}\right) .
$$

For $\epsilon \rightarrow 0$, i.e., when the argument of the scaling function is small, one expects qualitatively different behavior in the M-phase and on the DSM line. If the system has Dirac cones, the DOS is expected to be proportional to $|\epsilon|^{d-1}$ for $|\epsilon| \ll \epsilon_{0}$, so

$$
\rho(\epsilon) \sim \delta^{(d-z) \nu}\left(|\epsilon| \delta^{-z \nu}\right)^{d-1}=|\epsilon|^{d-1} \delta^{-(z-1) d \nu} .
$$

In the M-phase, on the other hand, the DOS is finite at $\epsilon=0$, and

$$
\rho(0) \sim \delta^{(d-z) \nu}\left(|\epsilon| \delta^{-z \nu}\right)^{0}=\delta^{(d-z) \nu} .
$$

Right at the tricritical point $\delta=0, \xi$ dependences in the prefactor and the argument of Eq. (8) should cancel, and consequently,

$$
\rho(\epsilon) \sim \delta^{(d-z) \nu}\left(|\epsilon| \delta^{-z \nu}\right)^{(d-z) / z}=|\epsilon|^{(d-z) / z} .
$$

Armed with the above observations, we next study the DOS numerically. First, the DOS at $\epsilon=0$ vanishes [Fig. 3(a)] around

$$
W_{\mathrm{c}}=6.4 \pm 0.1 \text {. }
$$
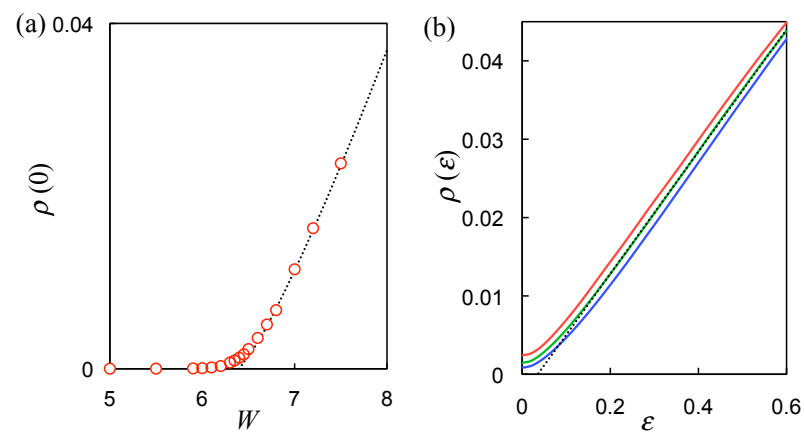

FIG. 3: (Color online) (a) The DOS at $\epsilon=0$. The point $W_{\text {c }}$ where $\rho(0) \rightarrow 0$ indicates the tricritical point $\mathbf{P}_{\mathrm{c}}$. (b) The DOSs around $W_{\mathrm{c}}$ (solid lines, $W=6.3,6.4,6.5$ from bottom to top). They can be approximated by a linear function (dotted line). The deviations for small energy regions are coming from the finite size effect $\rho(0) \sim L^{-2}$. We note that the effect of long ranged disorder [33], which might survive due to the finite lattice spacing, is not identified in our numerics.

We use this value to define $\delta$. The DOSs around $W=W_{\mathrm{c}}$, i.e., near $\mathbf{P}_{c}$, are plotted in Fig. 3(b). From the observed energy dependence and Eq. (13), we estimate

$$
\begin{aligned}
(3-z) / z & =1.00 \pm 0.15, \\
z & =1.5 \pm 0.1 .
\end{aligned}
$$

The result is consistent with the value $z=3 / 2$ obtained to the first order in the critical disorder strength in Ref. [6].

Next we derive the critical exponent $\nu$ from the DOS for small $|\epsilon|$. On the DSM line, by fitting the data to

$$
\rho(\epsilon) \sim c(\delta)|\epsilon|^{2},
$$

and then by fitting the coefficient $c(\delta)$ to the form

$$
c(\delta)^{-1} \sim \delta^{3(z-1) \nu_{\mathrm{DSM}}},
$$

we find [Fig. 4(a)]

$$
\begin{aligned}
3(z-1) \nu_{\mathrm{DSM}} & \simeq 1.16 \pm 0.05, \\
\therefore \nu_{\mathrm{DSM}} & \simeq 0.81 \pm 0.21 .
\end{aligned}
$$

The result can be interpreted physically as vanishing velocity of the Dirac electron along the DSM line towards the tricritical point $\delta=0$,

$$
v \sim \delta^{(z-1) \nu} \approx \delta^{0.4} .
$$

In the M-phase, on the other hand, by fitting the data to Eq. (12), we find [Fig. 4(b)]

$$
\begin{aligned}
(3-z) \nu_{\mathbf{M}} & \simeq 1.36 \pm 0.09, \\
\therefore \nu_{\mathbf{M}} & \simeq 0.92 \pm 0.13 .
\end{aligned}
$$

The values of $\nu_{\mathbf{M}}$ and $\nu_{\mathrm{DSM}}$ agree within the margin of error, and one expects in fact the same value on both sides 

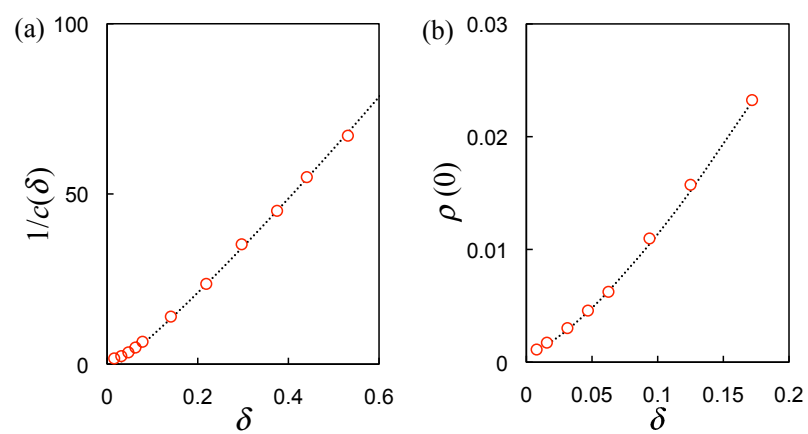

FIG. 4: (Color online) Dependence on $\delta$ (a) for Eq. (18) on the DSM line and (b) for Eq. (12) in the M-phase. We set $W_{\mathrm{c}}=6.4$.

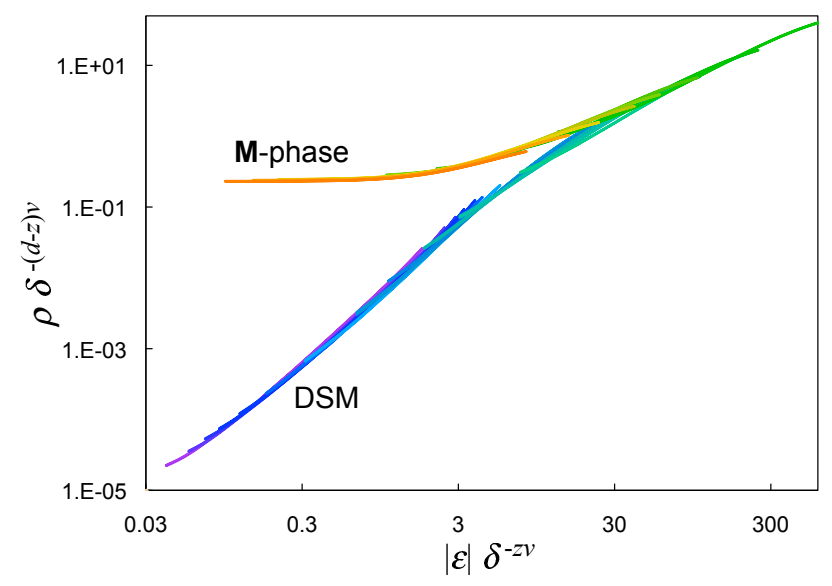

FIG. 5: (Color online) Single parameter scaling of the DOS. The upper branch corresponds to the DOS in M-phase, and the lower branch to the DSM line. We set the parameters $W_{\mathrm{c}}=6.4, z=1.5$, and $\nu=0.86=\left(\nu_{\mathrm{DSM}}+\nu_{\mathrm{M}}\right) / 2$.

of the transition. The first order perturbation theory in the location of the critical point [6] yields the characteristic $\nu_{\mathrm{DSM}}=\nu_{\mathrm{M}}=1$, which also falls within our intervals on both sides.

Lastly, and most importantly, we show that the single parameter scaling law, Eq. (10), fits successfully all of our numerical data. Figure 5 is the plot of the scaling combination $\rho(\epsilon) \delta^{-(d-z) \nu}$ vs. $|\epsilon| \delta^{-z \nu}$, with the above estimates of $W_{\mathrm{c}}, z$, and with using the average of the two exponents, $\nu=\left(\nu_{\mathrm{DSM}}+\nu_{\mathbf{M}}\right) / 2=0.86$. A similar value for $\nu$ would also follow had we solved Eqs. (19) and (22) under the assumption that $\nu_{\mathrm{M}}=\nu_{\mathrm{DSM}}$. After cutting off the relatively large energy region outside the Dirac cone and the very small energy region where the DOS becomes too small to estimate numerically, all the curves in Fig. 2 collapse onto two distinct branches, corresponding to the M-phase and to the DSM line, respectively. This is the central result of the present work.

The general scaling arguments imply interesting transport properties as well. Consider, for example, the wave packet dynamics 34]. We assume the mean square dis- placement $\left\langle\boldsymbol{r}^{2}(t, \epsilon)\right\rangle$ of the state with energy $\epsilon$ at time $t$, where $\langle\cdots\rangle$ represents both quantal and ensemble averages to be of the form

$$
\left\langle\boldsymbol{r}^{2}(t, \epsilon)\right\rangle \sim \xi^{2} g\left(t \xi^{-z},|\epsilon| \xi^{z}\right) .
$$

In the M-phase, one expects $\left\langle\boldsymbol{r}^{2}(t, \epsilon)\right\rangle=2 d D(\epsilon) t$ for large $t$ with $D(\epsilon)$ the diffusion coefficient at energy $\epsilon$. We focus only on the state with $\epsilon=0$,

$$
\left\langle\boldsymbol{r}^{2}(t, 0)\right\rangle \sim \xi^{2-z} t
$$

implying the diffusion coefficient $D(0)$ to diverge while the conductivity $\sigma(0) \sim \rho(0) D(0)$ to vanish towards $\mathbf{P}_{\mathrm{c}}$ as

$$
D(0) \sim \delta^{-(2-z) \nu}, \sigma(0) \sim \delta^{(d-2) \nu},
$$

the latter coinciding with the Wegner's relation 35], and predicts $\sigma(0) \sim \delta^{0.9}$. At $\mathbf{P}_{\mathrm{c}}$, the $\xi$ dependence should vanish, leading to

$$
\left\langle\boldsymbol{r}^{2}(t, 0)\right\rangle \sim \xi^{2}\left(t \xi^{-z}\right)^{2 / z}=t^{2 / z} \approx t^{1.3},
$$

which implies superdiffusion: when $z \simeq 1.5<2$, the system at $\mathbf{P}_{\mathrm{c}}$ is more diffusive than in the $\mathbf{M}$-phase. The numerical verification of such a superdiffusive behavior is, however, difficult, since we need to focus on the wave packet dynamics of $\epsilon=0$ state, the DOS of which is vanishing. Study is in progress to improve the situation.

Another interesting quantity is the conductance distribution along the DSM line. Away from $\mathbf{P}_{\mathrm{c}}$, the conductance will be narrowly distributed about the value expected in the absence of randomness as demonstrated in Ref. 22]. At $\mathbf{P}_{\mathrm{c}}$, we expect the scale independent broad conductance distribution as in the case of the Anderson transition [36, 37].

In summary, we have proposed the scaling of the density of states as a characteristic of the semimetal to metal transition in general, or, of the tricritical point among the two topologically different insulating phases and the metallic phase, in particular. In contrast to the conventional Anderson transitions, the density of states plays the role of the order parameter and shows the universal single-parameter scaling. This idea of using DOS to characterize DSM is also relevant in different systems such as the ones reported recently in Refs. 33 and 38]. Furthermore, we have estimated numerically the dynamical exponent $z \simeq 1.5$, which is clearly different from the conventional value $z=3$ [35] for the Anderson transition in 3D. The critical exponent of divergence of the length scale $\nu \simeq 0.9$ is less accurate, but it also seems rather far from the conventional value $\nu \simeq 1.35[39$ for the Anderson transition in 3D symplectic class. The poor inaccuracy of $\nu$ originates from the uncertainty of $W_{\mathrm{c}}$ and $z$. High precision estimate of $W_{\mathrm{c}}$ by different methods such as the transfer matrix [22] would improve the estimate. 
In this paper, we have focused on the phase boundary of the strong and weak topological insulators. The reason is practical; the DSM line and the phase boundary of metal to topological insulator phases intersect with a large angle, allowing us to pinpoint $\mathbf{P}_{\mathrm{c}}$ easily. For the phase boundary of the strong topological and ordinary insulators (STI/OI) [22], it is rather challenging to locate $\mathbf{P}_{\mathrm{c}}$, because the DSM line and the phase boundary of metal to insulator seem to intersect with a shallow angle. Because of the universal nature of critical phenomena, we expect similar scaling behavior with the same critical exponents for the semimetal to metal transition for STI/OI. On the other hand, different critical behavior is expected for the case of $\mathbb{Z}$ topological superconductor described by a Bogoliubov-de Gennes Hamiltonian, which shows similar phase diagram but belongs to a different universality class (DIII).

This work was supported by Grants-in-Aid for Scientific Research (C) (Grants No. 23540376) and Grants-inAid 24000013. I. F. H. is supported by the NSERC of Canada. K. K. and T. O. would like to thank Zhejiang Institute of Modern Physics, where fruitful discussion with V. E. Sacksteder and K. Slevin has been made.

[1] A. P. Schnyder, S. Ryu, A. Furusaki, and A. W. W. Ludwig, Phys. Rev. B 78, 195125 (2008).

[2] A. Kitaev, AIP Conference Proceedings 1134, 22 (2009).

[3] I. Herbut, A Modern Approach to Critical Phenomena (Cambridge University Press, Cambridge, 2007).

[4] R. Shindou and S. Murakami, Phys. Rev. B 79, 045321 (2009).

[5] E. Fradkin, Phys. Rev. B 33, 3263 (1986).

[6] P. Goswami and S. Chakravarty, Phys. Rev. Lett. 107, 196803 (2011).

[7] G. E. Volovik, The Universe in a Helium Droplet (Oxford University Press, 2003).

[8] A. A. Burkov and L. Balents, Phys. Rev. Lett. 107, 127205 (2011).

[9] A. A. Burkov, M. D. Hook, and L. Balents, Phys. Rev. B 84, 235126 (2011).

[10] M. Neupane, S.-Y. Xu, R. Sankar, N. Alidoust, G. Bian, C. Liu, I. Belopolski, T.-R. Chang, H.-T. Jeng, H. Lin, et al., arXiv:1309.7892 (2013).

[11] S. Borisenko, Q. Gibson, D. Evtushinsky, V. Zabolotnyy, B. Büchner, and R. J. Cava, arXiv:1309.7978 (2013).

[12] I. F. Herbut, Phys. Rev. Lett. 87, 137004 (2001).
[13] I. F. Herbut, V. Juričić, and O. Vafek, Phys. Rev. Lett. 100, 046403 (2008).

[14] P. W. Anderson, Phys. Rev. 109, 1492 (1958).

[15] B. Kramer and A. MacKinnon, Rep. Prog. Phys. 56, 1469 (1993).

[16] F. Evers and A. D. Mirlin, Rev. Mod. Phys. 80, 1355 (2008).

[17] We employed a polynomial of the second order for the extrapolation. The result is not sensitive to the extrapolation method near $\mathbf{P}_{\mathrm{c}}$.

[18] M. Z. Hasan and C. L. Kane, Rev. Mod. Phys. 82, 3045 (2010).

[19] L. Fu, C. L. Kane, and E. J. Mele, Phys. Rev. Lett. 98, 106803 (2007).

[20] J. E. Moore and L. Balents, Phys. Rev. B 75, 121306 (2007).

[21] R. Roy, Phys. Rev. B 79, 195322 (2009).

[22] K. Kobayashi, T. Ohtsuki, and K.-I. Imura, Phys. Rev. Lett. 110, 236803 (2013).

[23] S. M. Young, S. Zaheer, J. C. Y. Teo, C. L. Kane, E. J. Mele, and A. M. Rappe, Phys. Rev. Lett. 108, 140405 (2012).

[24] J. Li, R.-L. Chu, J. K. Jain, and S.-Q. Shen, Phys. Rev. Lett. 102, 136806 (2009).

[25] C. W. Groth, M. Wimmer, A. R. Akhmerov, J. Tworzydło, and C. W. J. Beenakker, Phys. Rev. Lett. 103, 196805 (2009).

[26] H.-M. Guo, G. Rosenberg, G. Refael, and M. Franz, Phys. Rev. Lett. 105, 216601 (2010).

[27] A. Yamakage, K. Nomura, K.-I. Imura, and Y. Kuramoto, J. Phys. Soc. of Jpn. 80, 053703 (2011).

[28] E. Prodan, Phys. Rev. B 83, 195119 (2011).

[29] A. Yamakage, K. Nomura, K.-I. Imura, and Y. Kuramoto, Phys. Rev. B 87, 205141 (2013).

[30] Q. Wu, L. Du, and V. E. Sacksteder, Phys. Rev. B 88, 045429 (2013).

[31] A. Weiße, G. Wellein, A. Alvermann, and H. Fehske, Rev. Mod. Phys. 78, 275 (2006).

[32] C.-X. Liu, X.-L. Qi, H.-J. Zhang, X. Dai, Z. Fang, and S.-C. Zhang, Phys. Rev. B 82, 045122 (2010).

[33] R. Nandkishore, D. A. Huse, and S. L. Sondhi, arXiv:1307.3252 (2013).

[34] T. Ohtsuki and T. Kawarabayashi, J. Phys. Soc. Jpn. 66, 314 (1997).

[35] F. Wegner, Z. Phys. B 25, 327 (1976).

[36] B. Shapiro, Phys. Rev. Lett. 65, 1510 (1990).

[37] K. Slevin and T. Ohtsuki, Phys. Rev. Lett. 78, 4083 (1997).

[38] Y. Ominato and M. Koshino, arXiv:1309.4206 (2013).

[39] Y. Asada, K. Slevin, and T. Ohtsuki, J. Phys. Soc. Jpn., Supplement 74, 258 (2005). 